\title{
CANTILEVER TYPE SWITCH DESIGN
}

\author{
Gajanan D.Patil $^{1}$, N. R. Kolhare ${ }^{2}$, Pradip Bhosale ${ }^{3}$, A.I.Rokade ${ }^{4}$ \\ ${ }^{I}$ Assistant Professor, Dept. of Electronics and Tele-Communication, PRMIT\&R, Amravati (M.S.), India \\ ${ }^{2}$ Assistant Professor, Dept. of Electronics and Tele-Communication, GECA, Aurangabad (M.S.), India \\ ${ }^{3}$ Assistant Professor, Dept. of Electronics and Tele-Communication, SGOI COE, BELHE, A.nagar (M.S.), India \\ ${ }^{4}$ Assistant Professor, Dept. of Electronics and Tele-Communication, PRMIT\&R, Amravati (M.S.), India
}

\begin{abstract}
This paper deals with the RF (Radio Frequency)-MEMS (Micro-Electro-Mechanical-System) switch design using the coventorware software and its superiority over the existing technologies like PIN Diodes and Field-Effect-Transistors regarding size, power, Isolation and Insertion loss, and graphically how Pull-in voltage affects on the deflection of the switch. Also this paper deals with the fabrication process of the cantilever switch.
\end{abstract}

Keywords: Etching, Sacrificial Layer, Actuation Voltage, Electrostatic Force, RF Switch, PIN Diodes, Stiction, Isolation and Insertion loss.

\section{INTRODUCTION}

RF MEMS Switches are used for make and break contact between the transmission lines, and operates in the frequency range $0.1 \mathrm{GHz}$ to $40 \mathrm{GHz}$. In the last two decades MEMS provided very strong and effective solution in the various passive circuits applications like varactors, switches and in various complex networks like filters.

As per recent generation requirements in terms device should be small in size, should be effective in performance such as low loss, less DC power consumption, less insertion loss and high isolation between the plates and for high frequency applications device should have high Q-factor. These all requirements full filled by the MEMS technology and practically implemented as effective technology for Military applications, mobile, wireless and satellite communications. The RF MEMS technology dominates over the existing technologies based devices like PIN diodes and FET. As per following characteristics mentioned in the Table 1.

MEMS Switches combines the advantages of Mechanical and Semiconductor Switches together and yields the advanced switches which will fulfill all the requirements like small in size, low DC power consumption, lower insertion loss, high off-state isolation [1]. Because of electromechanical isolation, without any losses RF circuit coupled significantly to the actuation circuit. Electromechanical coupling is done through electro statically through air less power required because power consumes only when actuation occurs.

Based on MEMS actuation, MEMS switches can be classified by following three characteristics;

i. RF circuit configuration,

ii. Mechanical structure,

iii. Form of contact.
Table -1: Comparison between various RF Switches

\begin{tabular}{|l|l|l|l|l|l|}
\hline $\begin{array}{l}\text { Switch } \\
\text { Type }\end{array}$ & $\begin{array}{l}\text { Isolatio } \\
\mathrm{n}\end{array}$ & $\begin{array}{l}\text { Insertion } \\
\text { Loss }\end{array}$ & $\begin{array}{l}\text { Power } \\
\text { Consu- } \\
\text { mption }\end{array}$ & $\begin{array}{l}\text { Switchi } \\
\text { ng Time }\end{array}$ & $\begin{array}{l}\text { Linea } \\
\text { rity }\end{array}$ \\
\hline $\begin{array}{l}\text { PIN } \\
\text { Diode }\end{array}$ & Medium & Good & More & Good & Poor \\
\hline FET's & Low & Good & Medium & $\begin{array}{l}\text { Excelle } \\
\text { nt }\end{array}$ & Poor \\
\hline $\begin{array}{l}\text { RF } \\
\text { MEMS }\end{array}$ & Large & Excellent & Less & Poor & $\begin{array}{l}\text { Excel } \\
\text { lent }\end{array}$ \\
\hline
\end{tabular}

Basically from a switch contact perspective, there are two types of switches based on the MEMS technology Application point of view- Series Contact Switches and Shunt Capacitive Switches.

\subsection{Series Contact Switches}

The basic structure of a MEMS contact series switch is its conductive beam suspended over the transmission line. Application of dc bias exerts an electrostatic force on the beam, which lowers the beam across the gap and shorts the open ends of the transmission line. After removing the dc bias, the spring restoring force in the beam returns it to its suspended (up) position.

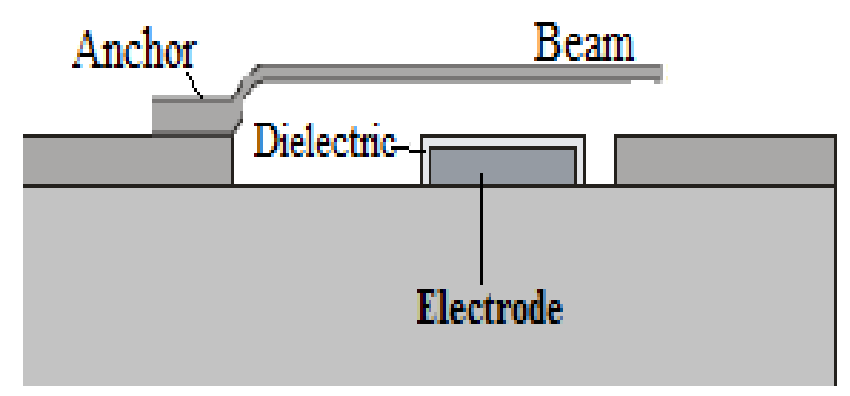

Fig -1: Series Contact Switch 


\subsection{Shunt Capacitive Switches}

In such switches grounded beam is suspended over a dielectric pad on the transmission line. When the beam is in up position, the capacitance of the line dielectric air beam is of the order of $\sim 50 \mathrm{pF}$, which translates to a high impedance path to ground through the beam. However when a dc voltage is applied between the transmission line and the electrode, the induced electrostatic force pulls down the beam which is to be coplanar with the dielectric pad results in lowering the capacitance to $\mathrm{pF}$ levels, impedance of the path through the beam for high frequency (RF) signal and shorting the RF to ground.

The capacitive switch insertion loss is lower than $1.2 \mathrm{~dB}$ up to $40 \mathrm{GHz}$, extracted up-state capacitance is $30 \mathrm{PF}$ and isolation is $1.3 \mathrm{~dB}$ at $1 \mathrm{GHz}, 26 \mathrm{~dB}$ at $20 \mathrm{GHz}$, and $27 \mathrm{~dB}$ at $40 \mathrm{GHz}$. Due to one end is free in cantilever type switch, it requires lower actuation voltage compared to Air bridge MEMS switch [9]. The capacitive MEMS switch is as shown in Figure 2.

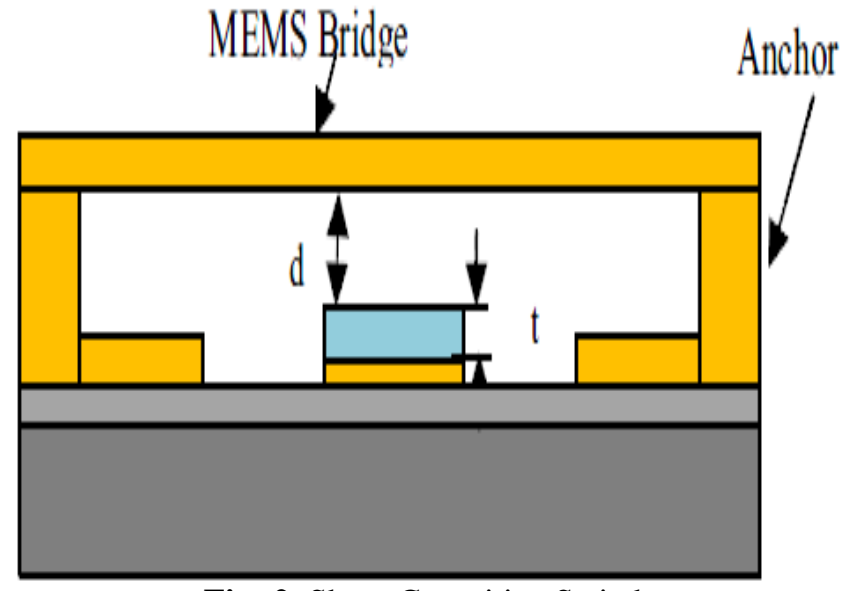

Fig -2: Shunt Capacitive Switch

\section{RF PERFORMANCE}

For designing cantilever beam as an actuator we must know the pull-in voltage, hold down voltage, spring constant required for cantilever and the resonant frequency for cantilever. Also needs to consider switching lifetime and switching time. The Solid structure of RF MEMS Cantilever Switch looks as shown in Figure 3(a) and its side view is shown in the Figure 3(b).

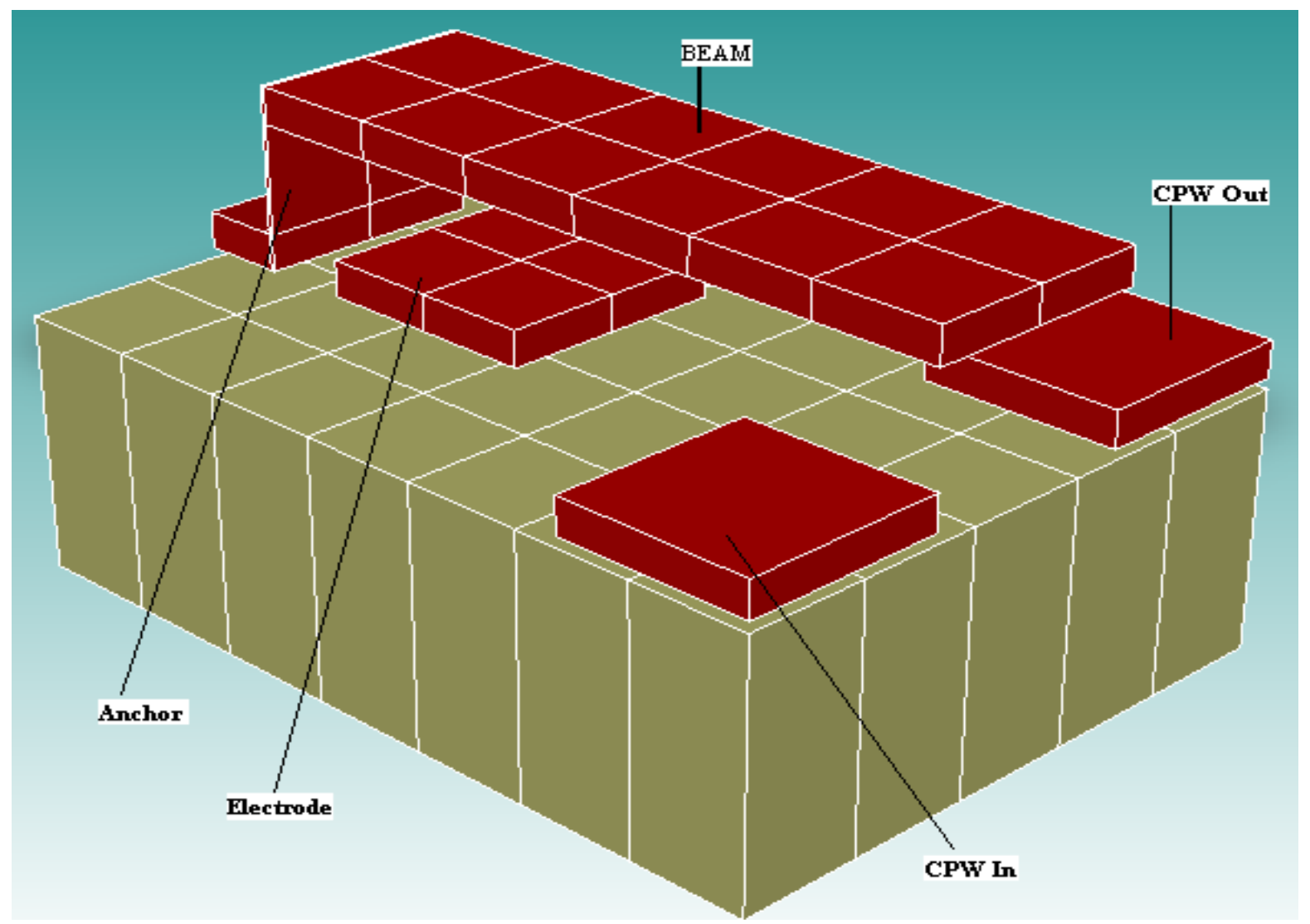

Fig -3(a): Solid Cantilever switch 


\subsection{Actuation Voltage}

The working of the cantilever switch is mainly depends on the applied electric field to the electrode $\mathrm{V}$, because of this applied field the electrostatic force $\mathrm{F}$ is exerted on the beam, this electrostatic force is measured as in equation (1),

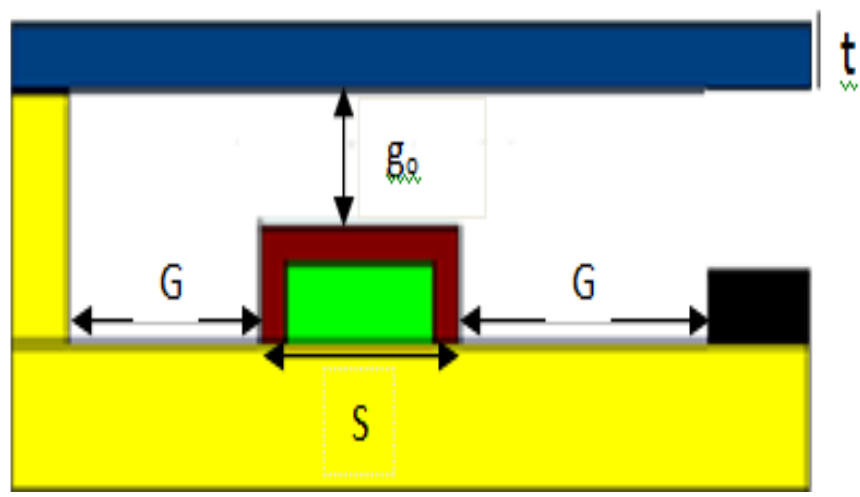

Fig -3(b): Side view of Cantilever switch

$$
F=\frac{\in_{0} A V^{2}}{2 g^{2}}, \mathrm{~F}_{\mathrm{r}} \propto \mathrm{K}_{\mathrm{t}} \cdot \Delta \mathrm{x}
$$

Where, $\mathrm{V}$ is applied voltage, $\mathrm{A}$ is contact area between electrode and beam \& $g$ is the deflected gap between electrode and the beam, and $\mathrm{K}_{\mathrm{t}}$ is the spring constant. When the applied voltage goes on increasing the electrostatic force exerted on the cantilever beam is goes on increasing which will tends to beam pull down towards the electrode tends to decreases the gap present between the beam and the electrode.

When this applied voltage goes on increases the time comes at certain value of applied voltage beyond that voltage the electrostatic force $\mathrm{F}$ is greater in magnitude than the restoring force which is exerted by the anchor because of the spring constant of the material. At applied voltage the electrostatic force dominates over the restoring force which pulls down beam towards the electrode as shown in Figure.7, which completes the path causes the signal to move from input to output. This makes the switch on. Here we designed the Cantilever switch having beam length 150 $\mu \mathrm{m}$, width of beam (W) $40 \mu \mathrm{m}, g_{0}$ is $2.2 \mu \mathrm{m}$ and $0.7 \mu \mathrm{m}$ thickness of the beam. Values of Pull in voltages by theoretical \& Coventorware simulation are $38.8 \mathrm{~V} \& 40 \mathrm{~V}$ respectively as shown in the Figure 5 . This voltage at which the beam pulled down towards the electrode is called as the pull-in voltage $\mathrm{V}_{\mathrm{p}}$ and is given by [4], [5] the equation (2) as,

$$
V_{p}=\sqrt{\frac{8 K_{t} g_{0}{ }^{3}}{27 \epsilon_{0} A}}
$$

Where $\mathrm{K}_{\mathrm{t}}$ is the spring constant of the beam material, $g_{0}$ is the original gap between the beam and the electrode and $\epsilon_{0}$ is the permittivity of the material. The spring constant is calculated by the equation (3) as,

$$
K_{t}=\frac{E W t^{3}}{4 l_{m}^{3}}
$$

Where, $\mathrm{E}$ is the young's modulus of the material, $\mathrm{t}$ is the thickness of the beam and $l_{m}$ is the enforced length of the beam is given by the equation (4) as,

$$
l_{m}=l-W-l_{s}
$$

Where $l$ the length of the beam, $\mathrm{W}$ is the width and $l_{s}$ is the isolation length of the beam.

Due to the applied voltage movement of the cantilever beam switch is as shown in the Figure 4.

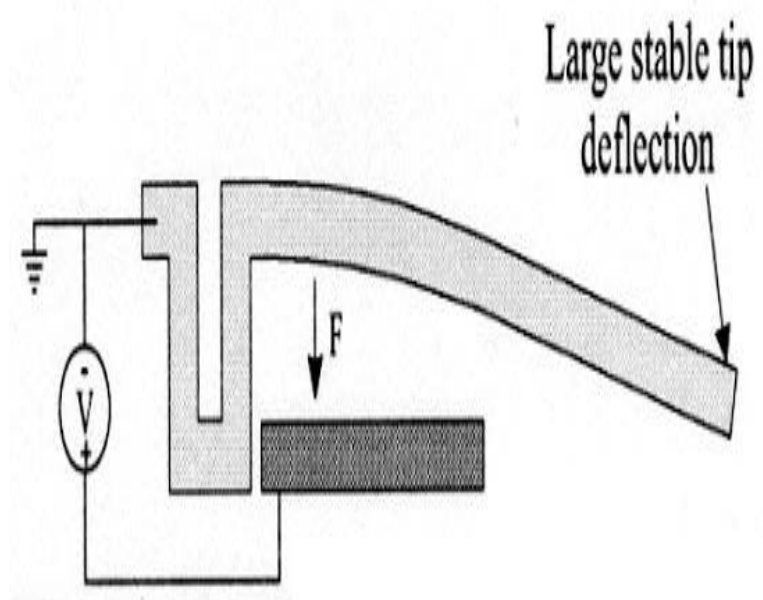

Fig -4: Movement of cantilever switch verses applied voltage

Initially as the applied voltage goes on increases the gap between the beam and ground electrode slowly reduces, as the gap becomes $2 / 3$ of original gap the beam suddenly pull downs. And the figure 5 represents the voltage required to pull down the cantilever beam to make switch as an on. The figure 6 shows the electrostatic force exerted by the applied voltage to the electrode.

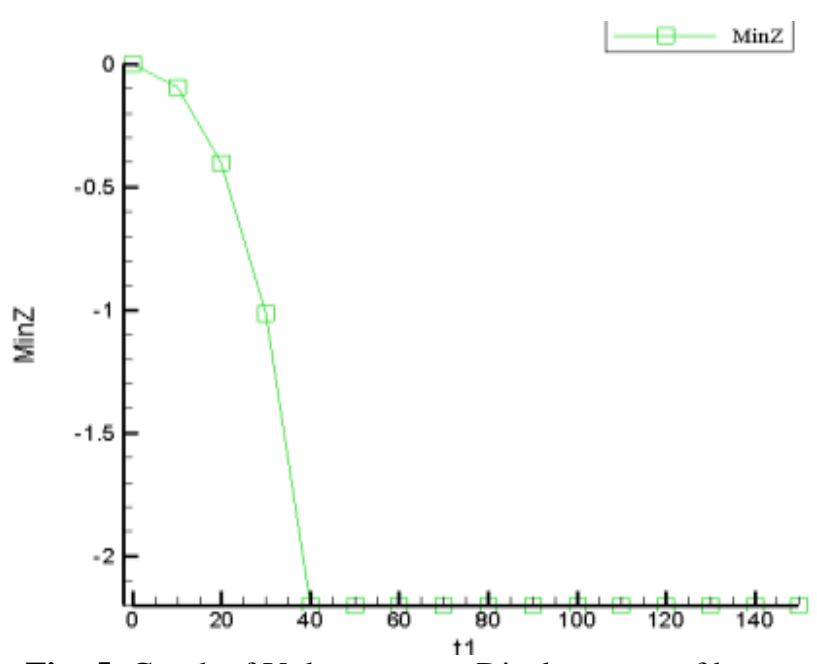

Fig -5: Graph of Voltage verses Displacement of beam 
Also from the different analysis on the structure it is observed that the actuation voltage required pulling the beam downward

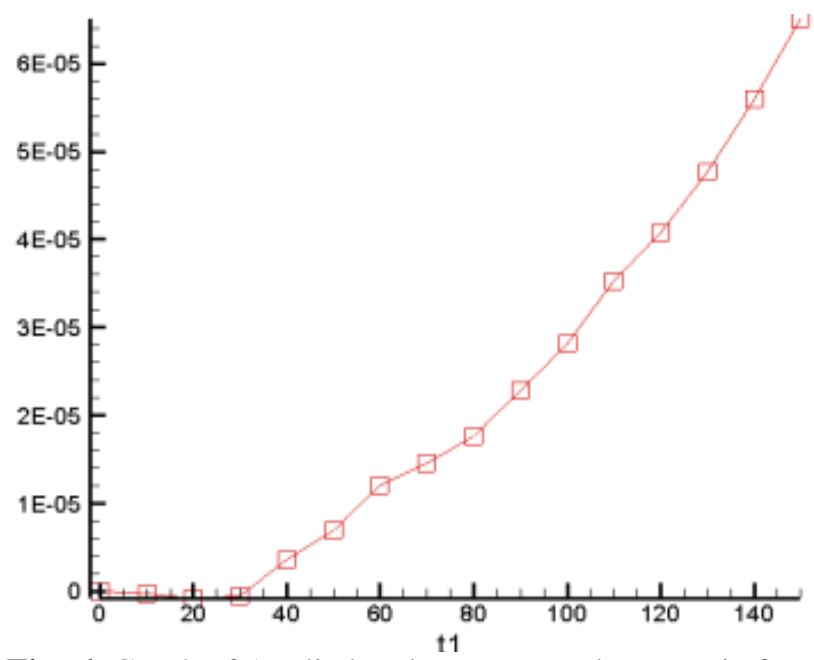

Fig -6: Graph of Applied Voltage verses electrostatic force on the beam

To make switch on is varies according to the length of the beam and also by Varying area of the actuated electrode. This is shown in the Table 2 .

Table -2: Shows Different Lengths Beam and the Required Pull in Voltage

\begin{tabular}{|l|l|}
\hline Length of Beam & Pull in Voltage $\mathrm{V}_{\mathrm{p}}$ \\
\hline $150 \mu \mathrm{m}$ & $40 \mathrm{~V}$ \\
\hline $180 \mu \mathrm{m}$ & $16 \mathrm{~V}$ \\
\hline $200 \mu \mathrm{m}$ & $11 \mathrm{~V}$ \\
\hline $250 \mu \mathrm{m}$ & $7 \mathrm{~V}$ \\
\hline $280 \mu \mathrm{m}$ & $5 \mathrm{~V}$ \\
\hline
\end{tabular}

\subsection{Switching Times}

Using 4-point resistance measurement and the laser vibrometer the electrical resistance and mechanical displacement of the switch is measured as a function of time. The closing time depends on actuation voltage whereas opening time depends on the mechanical properties of the switch by proper scaling the MEMS devices the switching time can be scale downs, as

$$
t_{s} \approx 3.67 \frac{V_{p}}{V_{s} \omega_{0}}, \quad f_{0}=\sqrt{\frac{K}{m}}
$$

Where $\left(\omega_{0}, f_{0}\right)$ is resonant frequency, $V_{p}$ is pull-in voltage and $V_{s}$ is applied voltage.

\section{FABRICATION PROCESS}

In the fabrication process of the cantilever switch is explained roughly step by step and Figure 3.1 Through 3.6 Explains Fabrication Process of Cantilever Type Switch, as bellow

\subsection{Substrate Creation}

To form the cantilever type structure we require the platform is called the substrate, silicon dioxide $\left(\mathrm{SiO}_{2}\right)$ material used as the substrate. In this design the substrate of $150 \mu \mathrm{m}$ length, $110 \mu \mathrm{m}$ width and $5 \mu \mathrm{m}$ thickness is used.

Fig -3.1: Substrate

\subsection{Electrode Formation}

Electrode is formed with the help of generic plasma enhanced chemical vapor deposition (PECVD) and generic dry etching process by exposing the part of the deposited electrode material through the openings in the masking layers and removes the unnecessary material. In this design Aluminum material is used for the electrode purpose, as shown in figure 3.2

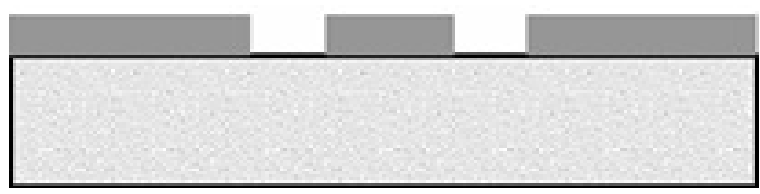

Fig -3.2: Actuated Electrode Formation

\subsection{Deposition of Silicon Nitride Layer}

To avoid the stiction problem, arises in the metal to metal contact occurs which affects on the smooth switching operation without this layer. In this design $0.2 \mu \mathrm{m}$ thick $\mathrm{SiN}_{3}$ layer is deposited over the electrode by PECVD process, as shown in figure 3.3.

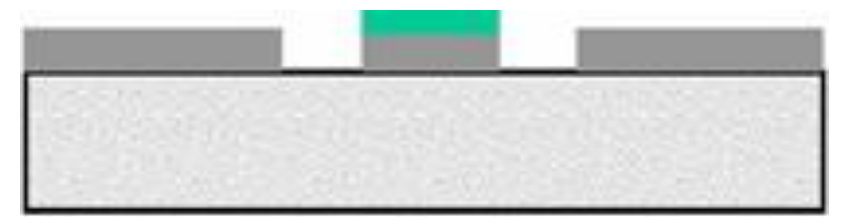

Fig -3.3: Deposition of $\mathrm{SiN}_{3}$

\subsection{Sacrificial Layer Formation}

To make the cantilever beam over the free space and separation between the CPW lines, BPSG material of 2.2 $\mu \mathrm{m}$ in height is used as shown in the figure 3.4

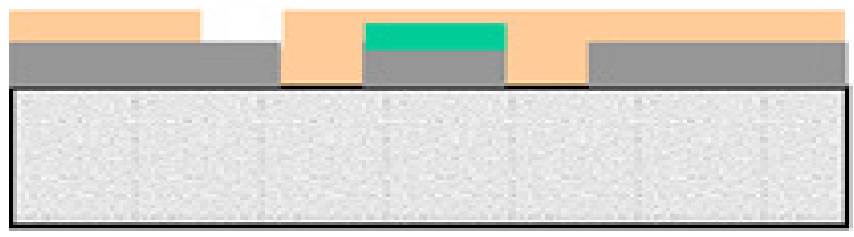

Fig -3.4: Deposition of Sacrificial Layer 


\subsection{Forming Cantilever Beam}

By using low chemical vapor deposition (LPCVD) $0.7 \mu \mathrm{m}$ thickness and $2.2 \mu \mathrm{m}$ hight Aluminum cantilever beam is formed, as shown in the figure 3.5

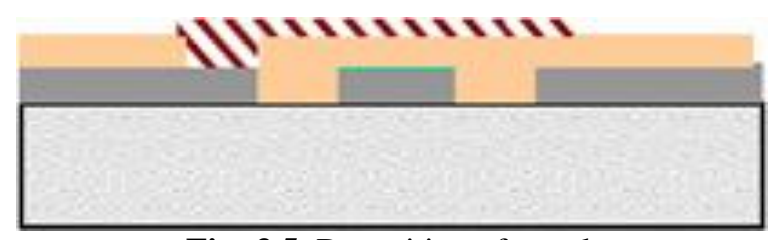

Fig -3.5: Deposition of metal

\subsection{Removing the Sacrificial Layer}

After cantilever beam structure formation the BPSG material is deleted to make the beam free in the air by using plasma etching process, as shown in figure 3.6

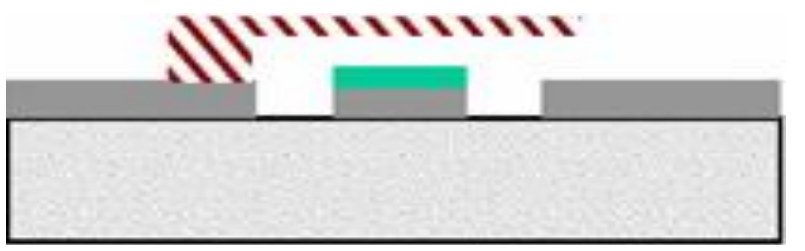

Fig -3.6: Deleting Sacrificial Layer

\section{CONCLUSION}

In this Paper we have seen how cantilever switch is designed and the different observations have been studied like varying the length of the beam we can reduce the pull in voltage required to make the switch on and off, also how can voltage relates with the electrode contact area and gap between the electrode and the actuating beam is seen in this paper. Also in this paper how fabrication can be done to prepare the RF switch is presented.

\section{REFERENCES}

[1]. P. D. Grant, R. R. Mansour"A Comparison Between RF MEMS Switches and Semiconductor Switches" in Can. J. Elect. Comput. Eng., Vol. 27, No. 1, pp. 33-39, Jan. 2002.

[2]. N. Charalampidis, D. Kampitaki"On the Design of an Ohmic RF MEMS Switch for reconfigurable microstrip antenna applications".

[3]. A. K. Sharma, C. G. Balaji, S. G. Singh, " Ohmic RF MEMS Switch With Low Loss And Low Force On Quartz For Reconfigurable Circuits," Vol. 3, Issue 1, pp. 45-54, Mar 2013.

[4]. Elmer S. Hung and Stephen D. Senturia, "Extending the Travel Range of Analog-Tuned Electrostatic Actuators, "Journal of Microelectro mechanical Systems, Vol. 8, No. 4, December 1999.

[5]. S. D. Senturia and P. M. Osterberg, "M-TEST: A test chip for MEMS material property measurement using electrostatically actuated structures, 'J. Microelectromech. Syst., vol. 6, no. 2, pp. 107-118, 1997.

[6]. J. I. Seeger and S. B. Crary, "Stabilization of electrostatically actuated mechanical devices," in Proc. Transducers '97, pp. 1133-1136.
[7]. Gabril M. Rebeiz, "RF MEMS: Theory, Design, and Technology,” John Wiley \& Sons, 2003.

[8]. Varadan V K, Vinoy K J and Jose K A, "RF MEMS and Their Applications, ” New York: Wiley, 2003.

[9]. E. R. Brown, "RF MEMS Switches for Reconfigurable Integrated Circuits", IEEE Transactions on Microwave Theory and Techniques, Vol. 46, no.11, November-1998.

[10]. R. Stefanini, M. Chatras, P. Blondy, Member, IEEE and G. M. Rebeiz, Fellow, "Miniature MEMS Switches for RF Application", Journal of Microelectromechanical Systems, Vol.20, No.6, December 2011.

[11]. S. N. Naduvinamani, B. G. Sheeparamatti, S. V. Kalalbandi, "Simulation of Cantilever Based RF-MEMS Switch Using Coventerware”, World Journal of Science and Technology 2011, 1(8): 149-153.

\section{BIOGRAPHIES}

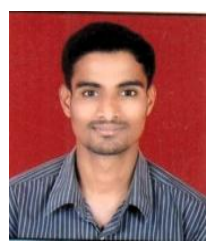

Gajanan D. Patil, M.E. in Electronics Engineering. Assistant Professor In Prof. Ram Meghe Institute Of Technology And Research Badnera - Amravati. Maharashtra.

Email: gajanan12385@gmail.com

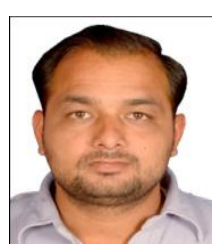

Pradip Bhosale, M.E. in Electronics Engineering. Assistant Professor SGOI COE, BELHE, The- Junner Dist-A.nagar, Maharashtra.

Email: pradipbhosale5@gmail.com

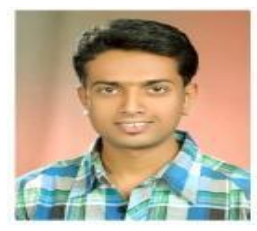

Ashay I. Rokade, M.Tech in Embeded System Engg. Assistant Professor In Prof. Ram Meghe Institute Of Technology And Research Badnera Amravati. Maharashtra.

Email: rokadeashay@gmail.com

Nilima R. Kolhare, Assistant Professor In Govt. College of Engineering, Aurangabad. Maharashtra.

Email: nilima_13@yahoo.com 